\title{
Intravitreal Injection of Bevacizumab for Retinopathy of Prematurity in an Infant with Peters Anomaly
}

\author{
Tsuyoshi Minami $^{a} \quad K^{2}$ azuki Kuniyoshi ${ }^{a} \quad$ Shunji Kusaka $^{c}$ Koji Sugioka ${ }^{a}$ \\ Hiroyuki Sakuramoto ${ }^{a}$ Masuo Sakamoto ${ }^{a}$ Akane Izu $^{b}$ Norihisa Wada ${ }^{b}$ \\ Yoshikazu Shimomura ${ }^{a}$ \\ Departments of ${ }^{a}$ Ophthalmology and ${ }^{b}$ Pediatrics, Kinki University Faculty of Medicine, and \\ 'Department of Ophthalmology, Sakai Hospital, Osaka, Japan
}

\section{Key Words}

Retinopathy of prematurity · Peters anomaly · Vascular endothelial growth factor · Anti-VEGF therapy · Bevacizumab

\begin{abstract}
Purpose: To report our findings in an infant with Peters anomaly type II whose retinopathy of prematurity (ROP) was treated with an anti-VEGF agent and surgeries. Case Report: A male infant weighing $548 \mathrm{~g}$ was born prematurely at 23 weeks and 1 day with corneal opacity and shallow anterior chambers in both eyes. At the postmenstrual age of 35 weeks and 3 days, the infant was tentatively diagnosed with stage 3 ROP because of a dilated tunica vasculosa lentis and ultrasonographic findings. The boy was treated with bilateral intravitreal injections of bevacizumab (IVB) because laser photocoagulation of the retina could not be performed due to the corneal opacity. The retina in the right eye detached 3 times, namely 5 days, 16 days, and 7 months after the IVB; encircling the scleral buckle and a vitrectomy with endolaser photocoagulation were therefore required. In his left eye, the retina was reattached after the initial IVB, and no additional treatment was required. ROP was not reactivated in both eyes until the last examination at the age of 2 years and 6 months. Conclusions: Our results showed that IVB is a useful treatment for ROP in patients with Peters anomaly. However, a retinal detachment can be a complication after IVB. The optimal timing of IVB for ROP in infants with hazy media needs to be determined.


Minami et al.: Intravitreal Injection of Bevacizumab for Retinopathy of Prematurity in an Infant with Peters Anomaly

\section{Introduction}

Peters anomaly is characterized by congenital corneal opacities, defects of the Descemet's membrane, shallow anterior chambers, cataracts, and glaucoma $[1,2]$. The corneal opacities in patients with Peters anomaly make the diagnosis and treatment of retinal disorders such as retinopathy of prematurity (ROP) difficult. Recently, the intravitreal injection of antivascular endothelial growth factors, including bevacizumab (IVB), was reported to be effective as a treatment for ROP $[3,4]$. One advantage of IVB over traditional treatments for ROP, e.g., retinal photocoagulation, is that it can be performed on infants with hazy media.

We present our findings in an infant with Peters anomaly accompanied by ROP that was treated with IVB. The research protocol was approved by the Ethics Review Board of the Kinki University Faculty of Medicine in November 2011, and the procedures conformed to the tenets of the Declaration of Helsinki of the World Medical Association.

\section{Case Report}

Our patient, a male infant, was born prematurely at 23 weeks and 1 day and weighed $546 \mathrm{~g}$. He was noted to have corneal opacities with shallow anterior chambers in both eyes soon after birth (fig. 1). He was diagnosed with Peters anomaly type II because he had corneal-lenticular adhesions in addition to a central corneal opacity with iridocorneal adhesions [1]. Details of the fundi could not be obtained because of the corneal opacity.

An ophthalmic examination performed at the postmenstrual age of 34 weeks and 2 days showed a dilated tunica vasculosa lentis, and ultrasonography showed an abnormal echo, which suggested ridge formation (white triangles in fig. 2). Although these findings suggested stage 3 ROP, which requires treatment [5], laser photocoagulation would have been difficult to perform because of the corneal opacity, dilated tunica vasculosa lentis, and the fixed pupils.

Thus, we selected to treat the eye with IVB $[3,4]$ (Avastin ${ }^{\circledR}$; Genentech Inc., South San Francisco, Calif., USA). A detailed clinical information and treatment options, including cryotherapy and IVB, were given to the family, and the parents agreed and signed an informed consent to proceed with the IVB.

At the postmenstrual age of 35 weeks and 3 days, $0.25 \mathrm{mg} / 0.01 \mathrm{ml}$ of bevacizumab were injected into the vitreous cavity of both eyes. Five days later, the dilatation of the tunica vasculosa lentis was reduced in both eyes, but a retinal detachment was detected by ultrasonography in the right eye (stage 4A ROP; black arrow in fig. 2). An encircling scleral buckle was placed on both eyes on the same day, and the retina of the right eye was detached again 11 days after the buckling surgery. We performed lens-sparing vitrectomy in the right eye because the tunica vasculosa lentis was reduced after the IVB and buckling surgery. The temporal area of the fundus, where the proliferation was most severe and the retina was partially detached, was barely observable, and vitrectomy with endolaser photocoagulation was performed. Since other areas of the fundus could not be observed due to the corneal opacity, we performed additional transscleral cryopexy in the right eye.

At 4 months after the IVB, a cataract with an increase of the intraocular pressure (IOP, $33 \mathrm{~mm} \mathrm{Hg}$ ) was detected in the right eye. The cataract was removed and the IOP was normalized. Seven months after the IVB, the corneal opacity was reduced in both eyes, allowing a better observation of the fundus (fig. 1, fig. 3). Ophthalmoscopy showed a third retinal detachment in the right eye (arrow in fig. 3), and fluorescein angiography showed an 
Minami et al.: Intravitreal Injection of Bevacizumab for Retinopathy of Prematurity in an Infant with Peters Anomaly

avascular zone in both eyes and some leakage of fluorescein in the right eye (fig. 3). The fovea of the right eye was displaced temporally (fig. 3).

A second vitrectomy was performed in the right eye, and no progression or reactivation of the ROP or retinal detachment was noticed at the last examination at the age of 2 years and 9 months. In his left eye, the IOP was normal and the retina was attached after the initial IVB, and no additional treatment was required. No apparent systemic side effects of the IVB were observed.

\section{Discussion}

The treatment for ROP in infants with corneal opacity is challenging. Although partial corneal opacity, which is occasionally observed in neonatal infants, allows clinicians to observe and treat ROP, dense and bilateral corneal opacities, which were present in our patient, obstructed the detection and treatment of the ROP.

We had several treatment options for our patient: IVB, combination of penetrating keratoplasty with laser photocoagulation, and endoscopic vitrectomy with laser photocoagulation. We selected IVB as the first treatment because it could be performed even in an eye with a corneal opacity. Penetrating keratoplasty was not considered before the treatment for ROP because we knew the results are not favorable in patients with Peters anomaly type II [2].

There are 3 problems in managing ROP in eyes with dense and bilateral corneal opacities: (1) how do we detect ROP, (2) when do we treat ROP, and (3) how do we treat ROP.

According to the results of the Early Treatment for Retinopathy of Prematurity (ETROP) study [5], the treatment for ROP should be performed within $72 \mathrm{~h}$ after a diagnosis of type 1 ROP. The most important finding required to make a decision on the treatment of the ROP is the 'plus disease' condition [5], which is difficult to recognize in infants with hazy media. Therefore, clinicians need to decide on the treatment for ROP in infants with hazy media without any information regarding the status of the fundus.

The ETROP Cooperative Group also reported that stage 3 ROP was present in $48.1 \%$ of infants whose birth weight was $<750 \mathrm{~g}$ and in $43.3 \%$ of infants whose gestational age was $\leq 27$ weeks [6]. In our institution, the first treatment is performed between the postmenstrual age of 30 and 35 weeks [7]. From the results of these treatments, the infants who had a high risk for ROP, mainly those with a gestational age of $\leq 27$ weeks or a birth weight of $<750$ $\mathrm{g}$, were recommended to be treated between the postmenstrual age of 30 and 35 weeks.

Our results indicated that IVB appeared to be effective and led to a regression of the ROP; however, additional surgeries were needed for the tractional retinal detachments. Although a retrospective analysis of our findings indicated that earlier IVB treatments may not lead to retinal detachments, the systemic condition of our patient did not allow an earlier treatment for the ROP.

A late reactivation of ROP [4, 8] as well as incomplete vascularization after IVB [4, 9] have recently been reported. The results of fluorescein angiography in our patient suggested that the ROP had not completely regressed even 7 months after the IVB (fig. 3). Longitudinal and careful observations are needed after IVB treatment for ROP.

In conclusion, IVB can be a treatment for ROP in infants with Peters anomaly. However, the diagnosis of ROP and the timing of the IVB in infants with hazy media need more investigation. 
Minami et al.: Intravitreal Injection of Bevacizumab for Retinopathy of Prematurity in an Infant with Peters Anomaly

\section{Acknowledgements}

The authors express gratitude to Kyutaro Ito, MD, working at the Department of Ophthalmology, Tottori Prefectural Central Hospital, for referring the patient to our clinic. We also thank Masahiko Fukuda, MD, and Chota Matsumoto, MD, in the Department of Ophthalmology, Kinki University Faculty of Medicine, for their suggestions regarding this paper.

This research was supported by the research grants to S.K. and K.K. from the Japan Society for the Promotion of Science, Japan (23592597) and to Y.S. from the Ministry of Health, Labour and Welfare, Japan (82259921).

\section{Disclosure Statement}

The authors have no proprietary or commercial interests related to this research.

\section{References}

$\longrightarrow$ Zaidman GW, Flanagan JK, Furey CC: Long-term visual prognosis in children after corneal transplant surgery for Peters anomaly type I. Am J Ophthalmol 2007;144:104-108.

$\longrightarrow 2$ Bhandari R, Ferri S, Whittaker B, Liu M, Lazzaro DR: Peters anomaly: review of the literature. Cornea 2011;30:939-944.

-3 Mintz-Hittner HA, Kennedy KA, Chuang AZ; the BEAT-ROP Cooperative Group: Efficacy of intravitreal bevacizumab for stage 3+ retinopathy of prematurity. N Engl J Med 2011;364:603-615.

-4 Kuniyoshi K, Sugioka K, Sakuramoto H, Kusaka S, Wada N, Shimomura Y: Intravitreal injection of bevacizumab for retinopathy of prematurity. Jpn J Ophthalmol 2014;58:237-243.

5 Early Treatment for Retinopathy of Prematurity Cooperative Group: Revised indications for the treatment of retinopathy of prematurity. Arch Ophthalmol 2003;121:1684-1696.

-6 Early Treatment for Retinopathy of Prematurity Cooperative Group: The incidence and course of retinopathy of prematurity: findings from the Early Treatment for Retinopathy of Prematurity study. Pediatrics 2005;116:15-23.

7 Kayazawa M, Kuniyoshi K, Sugioka K, Tsujioka D, Abe K, Matsumoto C, Wada N, Shimomura Y: Review of retinopathy of prematurity in very low birth weight infants and extremely low weight infants. Folia Jpn Ophthalmol Clin (Ganka Rinsho Kiyo) 2011;4:448-454.

-8 Hu J, Blair MP, Shapiro MJ, Lichtenstein SJ, Galasso JM, Kapur R: Reactivation of retinopathy of prematurity after bevacizumab injection. Arch Ophthalmol 2012;130:1000-1006.

-9 Tahija SG, Hersetyati R, Lam GC, Kusaka S, McMenamin PG: Fluorescein angiographic observations of peripheral retinal vessel growth in infants after intravitreal injection of bevacizumab as sole therapy for zone I and posterior zone II retinopathy of prematurity. Br J Ophthalmol 2014;98:507-512. 


\section{Case Reports in \\ Ophthalmology}

\begin{tabular}{l|l}
\hline Case Rep Ophthalmol 2014;5:318-324 \\
\hline DOI: 10.1159/000368298 & $\begin{array}{l}\text { ○ 2014 S. Karger AG, Basel } \\
\text { www.karger.com/cop }\end{array}$ \\
\hline
\end{tabular}

Minami et al: Intravitreal Injection of Bevacizumab for Retinopathy of Prematurity in an Infant with Peters Anomaly

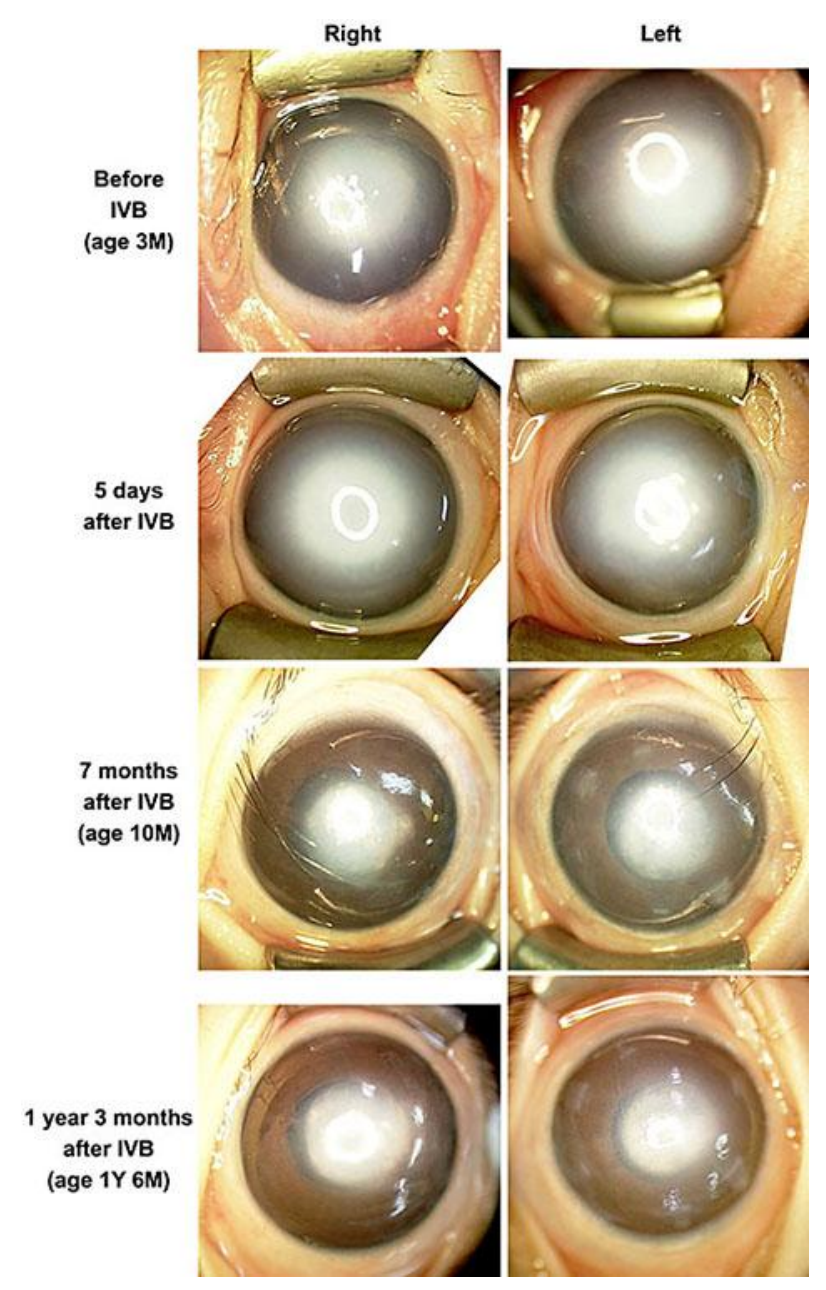

Fig. 1. Photographs of the anterior segment of eyes with Peters anomaly type II and ROP. The dense corneal opacities are gradually reduced. These photographs were taken with a RetCam ${ }^{\circledR} 3$ (Clarity Medical Systems, Pleasanton, Calif., USA). Y=Year; $\mathrm{M}=$ month. 
Case Reports in

Ophthalmology

\begin{tabular}{l|l}
\hline Case Rep Ophthalmol 2014;5:318-324 \\
\hline DOI: 10.1159/000368298 & $\begin{array}{l}\text { ○ 2014 S. Karger AG, Basel } \\
\text { www.karger.com/cop }\end{array}$ \\
\hline
\end{tabular}

Minami et al.: Intravitreal Injection of Bevacizumab for Retinopathy of Prematurity in an Infant with Peters Anomaly

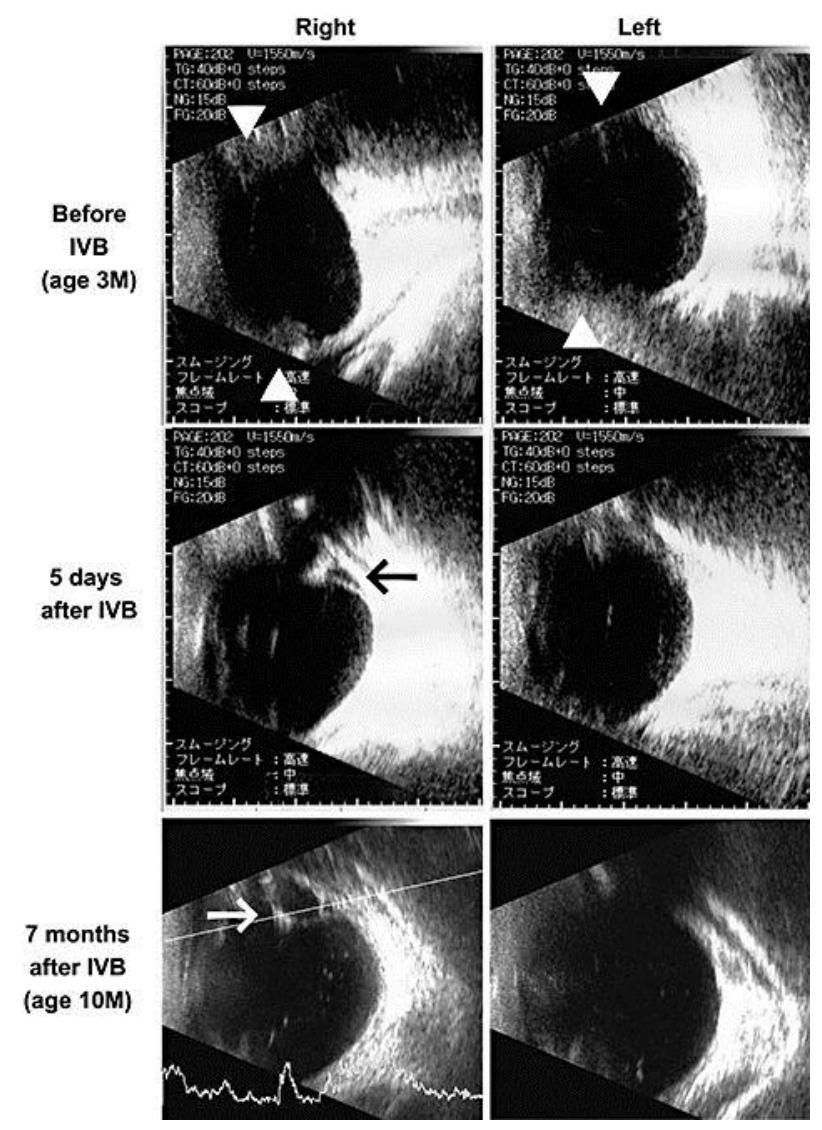

Fig. 2. Ultrasonographic findings of eyes with Peters anomaly and ROP. Before the IVB, abnormal echo was detected which suggested ridge formation (triangles in the upper row, stage 3 ROP). Five days after the IVB, a retinal detachment was suspected in the right eye (black arrow in the middle row, stage $4 \mathrm{~A}$ ROP). Seven months after the IVB, a third retinal detachment is suspected in the right eye (white arrow in the lower row, stage $4 \mathrm{~A}$ ROP). 
Case Reports in

Ophthalmology

\begin{tabular}{l|l}
\hline \multicolumn{2}{l}{ Case Rep Ophthalmol 2014;5:318-324 } \\
\hline DOI: 10.1159/000368298 & $\begin{array}{l}\text { ○ 2014 S. Karger AG, Basel } \\
\text { www.karger.com/cop }\end{array}$ \\
\hline
\end{tabular}

Minami et al.: Intravitreal Injection of Bevacizumab for Retinopathy of Prematurity in an Infant with Peters Anomaly

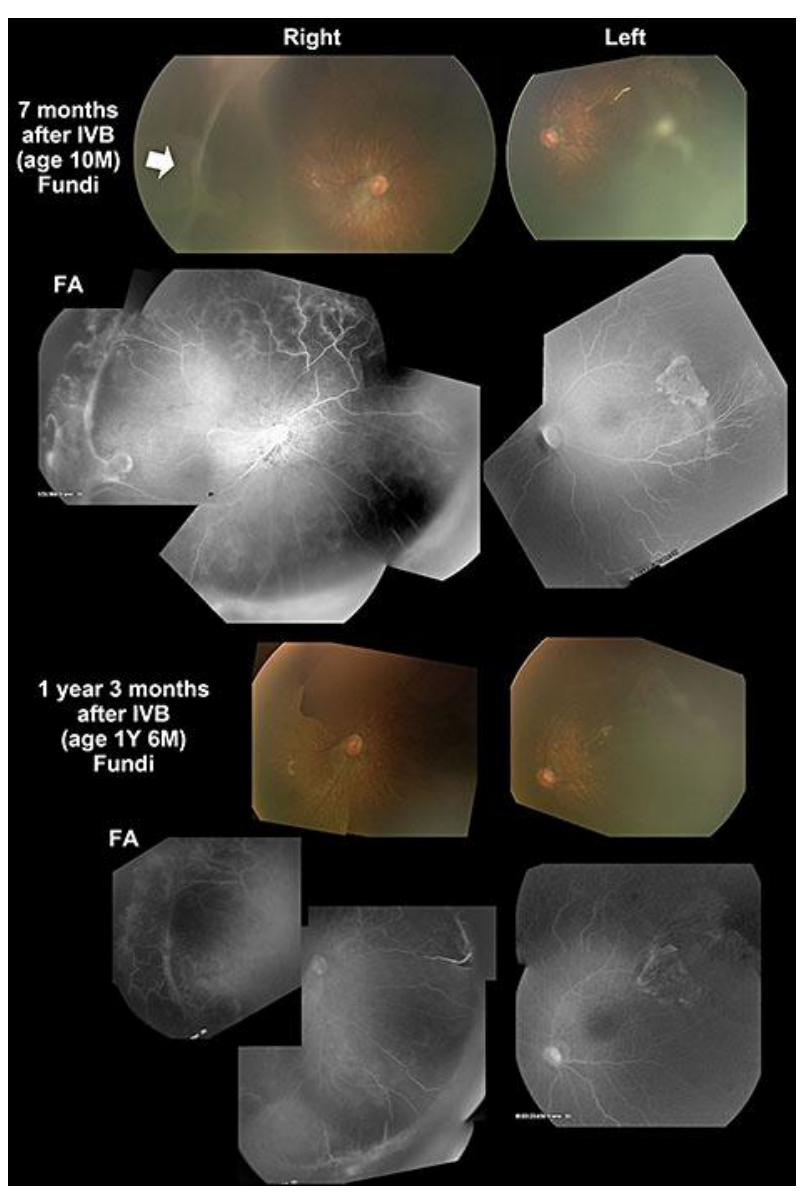

Fig. 3. Fundus photographs and fluorescein angiograms. A retinal detachment can be seen in the right eye 7 months after the IVB (arrow). An avascular zone and some leakage of fluorescein are still present in the periphery 1 year and 3 months after IVB. Photography was difficult because of the residual corneal opacity in both eyes. Fundus photography and fluorescein angiograms were performed using $\operatorname{RetCam}^{\circledR} 3$. 